\title{
Practical aspects of telehealth: set-up and preparation for video consultations
}

\author{
D. T. Allen, ${ }^{1,2}$ P. Caldwell, ${ }^{5,6}$ P. A. Komesaroff, ${ }^{8,9}$ P. K. Loh, ${ }^{13}$ R. Mozer, ${ }^{7}$ S. Sabesan, ${ }^{14,15}$ N. Shaheen, ${ }^{3}$ \\ P. Talman, ${ }^{10,11,12}$ M. Williams, ${ }^{16}$ and O. Grabinski ${ }^{4}$ on behalf of The Royal Australasian College of Physicians \\ Telehealth Working Group
}

${ }^{1}$ University of New South Wales, ${ }^{2}$ Quality Occupational Health, ${ }^{3}$ Aged Care Services, Royal North Shore Hospital, ${ }^{4}$ Policy and Advocacy unit, The Royal Australasian College of Physicians, ${ }^{5}$ Discipline of Paediatrics and Child Health and ${ }^{6}$ Centre for Kidney Research, The Children's Hospital at Westmead, Sydney, ${ }^{7}$ Rehabilitation Medicine, Rankin Park Centre, Newcastle, New South Wales, ${ }^{8}$ Faculty of Medicine, ${ }^{9}$ Monash Centre for the Study of Ethics in Medicine and Society and ${ }^{10}$ Department of Epidemiology and Preventative Medicine, Monash University, Melbourne, ${ }^{11}$ Neurosciences Department, Geelong Hospital, ${ }^{12}$ Deakin Medical School, Geelong, Victoria, ${ }^{13}$ Department of Geriatric Medicine, Royal Perth Hospital, Perth, Western Australia, ${ }^{14} \mathrm{School}$ of Medicine and Dentistry, James Cook University, ${ }^{15}$ Department of Medical Oncology, Townsville Cancer Centre, Townsville, and ${ }^{16}$ Department of Child and Adolescent Health, Mackay Hospital, Mackay, Queensland, Australia

\section{Key words}

telehealth, videoconference consultation, telemedicine.

\section{Correspondence}

Odette Grabinski, Policy and Advocacy Unit, The Royal Australasian College of Physicians, Sydney, NSW 2000, Australia.

Email: telehealth@racp.edu.au

Received 18 July 2013; accepted 4 August

2013.

doi:10.1111/imj.12264

\begin{abstract}
The third in a series of articles about the practical aspects of telehealth, this paper gives guidance on suitable setup for video consultations, including layout of rooms, managing sound and image quality, scheduling, testing and best practice in telehealth videoconferencing.
\end{abstract}

\section{Introduction}

The emphasis in this article is on practical guidance. In different specialties the requirements may vary, but the same general principles apply to all. Just like face-to-face consultations, video consultations are synchronous, that is they occur in real time. The obvious difference between a video consultation and a face-to-face consultation is that a full physical examination cannot be undertaken by the specialist. Therefore, any examination is either visual or done with the aid of an assistant or support person at the remote site. When approaching video consultations, it may be necessary to modify the extent of the history taking and/or to obtain assistance at the patient end. This means that you need to consider what information you need from the patient, from the referring general practitioner (GP) and to what degree the physical examination

Funding: None.

Conflict of interest: None. is needed or can be accomplished with the GP or other supporting healthcare professional.

When selecting suitable patients for telehealth consideration should be given to:

- The presenting problem and whether it is likely to be suitable for a telehealth consultation

- The urgency of the case

- The distance the patient may have to travel for a faceto-face consultation and the cost to the patient and risks to which he or she may be exposed

- The GP's recommendations on suitability and patient preference

- The availability of a suitable host site at the patient end (including in the patient's home).

The benefits of telehealth for patients include reduced travel and more timely service, with health outcomes at least equal to traditional face-to-face consultations. ${ }^{1}$ Telehealth does not, of course, preclude later face-to-face consultations. Telehealth consultations may be used to gather information prior to seeing the patient in person. 


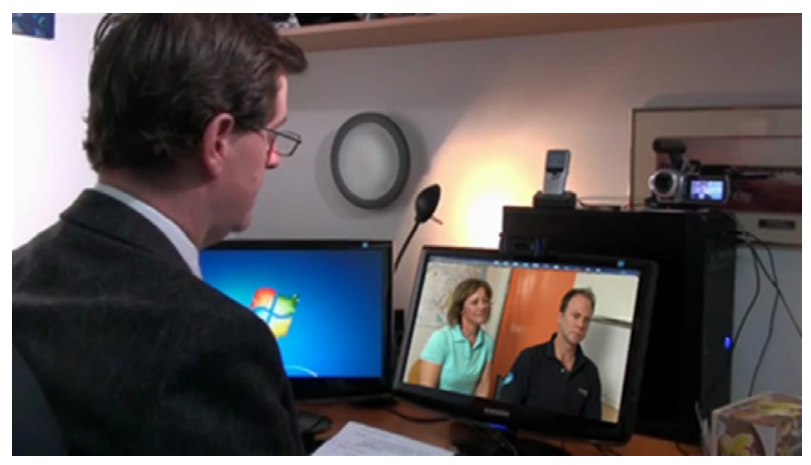

Figure 1 Illumination from behind the monitor.

Such an approach can minimise the risk of a patient not having adequate investigations or results available prior to the face-to-face consultation. A video consultation may also enable the physician to assess the urgency of the need for a face-to-face consultation, thereby facilitating an effective process of triage with minimal inconvenience to the patient. As healthcare providers become familiar with the processes, video consultations can be fitted easily into everyday schedules.

\section{Room layout}

Most physicians are comfortable with an older and more common form of telehealth: providing advice to patients over the telephone. Video consultations are really just an extension of this, with the added ability to visualise the patient, undertake a fuller and more reliable assessment, and therefore make better judgments and provide more complete information. In Australia, they are also reimbursable under the Medicare system. ${ }^{2}$

The video consultation will generally take place in a consulting or treatment room. The location chosen will depend on convenience and access to Internet connectivity. It is necessary to have access to a computer with a monitor and a good quality webcam. A wired connection to the Internet is preferable because wireless fidelity (Wi-Fi) and wireless Internet are less stable and more prone to interference.

Having a lamp positioned near the monitor, as in Figure 1, will enable the patient to see the specialist clearly. If the light source in the room is behind the specialist, then their face will be poorly illuminated and dark; this can lead to a poor patient experience.

The background should be simple and plain and without distracting images or objects. Most videoconferencing applications allow healthcare providers to see themselves on the screen so that they are aware of what the patient is seeing; some doctors find this distracting; however, this function can be switched off once you are familiar with it. Also, it is useful for the specialist to sit on a movable chair to enable them to remain in the middle of the screen.

The webcam should be positioned as close as possible to the top of the monitor, which generally sits at or just below eye level. The image of the patient on the screen should be positioned as close as possible to the webcam. ${ }^{3}$ Generally, when people are videoconferencing, they do not look directly at the webcam but rather at the other person's face on the monitor. During a telehealth consultation, if the latter image is far from the webcam lens, the patient may feel that you are not looking directly at them. Moving the patient's image on the monitor to the top of the screen close to the webcam can help create a sense of natural eye contact. If access is required to pathology or medical imaging results, this is best provided on a viewing monitor separate from the video conferencing monitor. As in Figure 1, two monitors may be used - one for videoconferencing and another for results or electronic health records.

To avoid unnecessary interruptions, consider locking the door or placing a sign on it indicating that a video consultation is underway. It may not be needed once practice staff, clinicians and patients become more familiar with video consultations. Also, consider minimising background noise if possible as this is distracting and can affect communication.

At the start of a video consultation, everyone should be identified. This minimises the risk of sensitive information being disclosed to unknown individuals not in the field of view of the webcam. It is important also to clarify who is present at the patient end, whether it is in a healthcare provider's office or in the patient's home.

\section{Sound quality}

While it is important for the specialist and the patient to be able to see each other, it is worth remembering that the consultation is primarily a conversation. Seeing the consultant's face in addition to hearing their voice certainly increases patient confidence, ${ }^{4}$ but a large proportion of any consultation consists of talking, and as such sound quality is crucial. The vast majority of video consultations carried out in Australia use Internet and computer technology. Accordingly, care should be taken to choose a system that provides adequate sound quality.

Making initial contact by conventional telephone and then switching on video when the consultation commences may facilitate continuity of the audio stream and reduce disruption should the sound drop out. From a practical point of view, an initial phone call to the patient end may in any case be helpful to ensure that the patient is ready. For example, if the sound quality of the video 
link is deficient, the telephone call can continue throughout the video consultation, and the audio option on the videoconferencing application can be left off. Most modern telephone handsets have hands-free mode, and generally the microphones are quite good at picking up voice. An effective video consultation is impossible if participants cannot hear each other clearly.

If the videoconferencing application and equipment provide reasonable quality audio with minimal dropouts, then they can be used as the main audio stream. Echo cancelling microphones (often integrated into quality webcams), personal speaker phones and iPod-type earphones can also improve sound quality.

\section{Webcam}

Webcams can be built into computers, viewing screens or peripheral devices connected to computers. Most will autofocus to macro distances to enable close up examination. Conventional webcams connected by universal serial bus (USB) can easily be repositioned with simple instruction to the patient or their assistant or patient end healthcare provider. They can be placed on a desk for close-up examination but need to be kept still to ensure that the image is clear and the autofocus can work effectively. Pan/tilt/zoom cameras allow panning from side to side, tilting up and down, or zooming in or out. While this provides extra utility, it does add complexity and expense. Most people choose to start with a conventional webcam, and if at a later date they feel the need to add more control, they can do so.

\section{Assistance at the patient end}

A nurse, Aboriginal health worker or GP may be present at the patient end during the consultation. While not essential, a support person or assistant enables at least some degree of physical examination during the consultation if needed. Increasing availability of devices such as digital stethoscopes (which allow auscultation at a distance), digital otoscopes and portable ultrasound machines will allow more detailed examinations to be undertaken. Nonetheless, for those starting telehealth consultations for the first time, a simple video consultation with the help of an assistant for a physical examination if needed is probably preferable. Extra devices can add complexity and may actually impede communication. If needed, a GP can make contact with 'Medicare Locals' for additional technical support and advice. ${ }^{5}$

\section{Familiarisation and troubleshooting}

Many people will be familiar with the problems of using technology and, while these days it is much more robust and easy to manage, occasionally problems will occur. It is worthwhile explaining to the patient at the outset of a telehealth consultation that should technical problems occur, the call will be continued by telephone until the video can be restarted or corrected. This will reduce patient anxiety should such a disruption occur. With experience, users will become familiar with the stability of the system they are using and, with a little practice, will be able to undertake basic troubleshooting themselves.

If the Internet connection is slow, some videoconferencing applications will allow the user to slow the frame rate to reduce the number of video frames per second. This helps to maintain a higher resolution image that can be particularly useful if a more detailed visual examination is required.

When image quality is poor, slowing the consultation and allowing more time with the patient will also help to minimise concerns. Telehealth consultations save patients time and expense $e^{1,6}$ and reduce inconvenience. Allowing a little extra time to ensure that adequate information is obtained can be reassuring for the patient.

\section{Case history}

A 56-year-old man, accompanied by his GP and his wife, is being reviewed by a haematologist from a tertiary centre. During the consultation, both ends can see but cannot hear each other. There is no real-time support service available because they are using free video conferencing software on the Internet.

\section{Discussion}

Solutions to this problem include continuing the consultation using hands-free telephones together with the images on the video monitors. Feedback should be sent to the software provider so deficiencies in the software can be addressed. If needed, hardware providers may assist with overcoming problems at either end.

\section{Testing}

Equipment should be tested regularly and prior to video consultations. It is helpful to initiate the connection 5 or 10 min before the agreed appointment time to ensure that everything is in order and the patient is ready. At least one practice staff member should be sufficiently familiar with the technology to test the system in advance of the consultation to solve minor technical problems. Once confidence in the stability of the system develops, less frequent testing will be needed, and 
support staff can assume responsibility for managing connections and ensuring the smooth functioning of e-clinics with minimal disruption.

\section{Scheduling}

Scheduling can be arranged either through the booking systems usually employed by the practice or by online systems accessed by both parties. Existing booking or appointment systems are often adequate - and may be more straightforward and convenient for patients unless the number of consultations is large. Several proprietary online booking systems are available.

\section{Medical records}

When first starting telehealth consultations, it is reasonable to continue to use existing record-keeping and correspondence systems. This will minimise any added complexity while familiarity with new technologies is being developed. Care should be taken to provide copies of correspondence to the patients' GP and referring healthcare provider. If the consultation is conducted in a rural setting, copies of correspondence for the hospital medical records might also be helpful to maintain continuity in case of frequent locums. With time, shared electronic records may be introduced to simplify exchange of information. It is important that all histories record the patient's consent to participate in telehealth consultations.

\section{Conclusion}

With simple preparation, testing and practice telehealth care delivery can generally be delivered efficiently and effectively. The possibility of problems before or during videoconferences is always possible, which can be minimised by a little extra care taken in the choice of both hardware and software support. The time spent on preparation and practice can be of enormous benefit to selected patients and can provide health outcomes comparable with face-to-face care and save significant travel time and inconvenience.

\section{References}

1 Wade VA, Karnon J, Elshaug AG, Hiller JE. A systematic review of economic analyses of telehealth services using real time video communication. BMC Health Serv Res 2010; 10: 233.

2 Specialist video consultations under Medicare. [MBS Online page on the internet]. Canberra: Department of Health and Ageing. [cited 2013 May]. Available from URL: http://www .mbsonline.gov.au/telehealth
3 The Physicians Telehealth Support Project. [RACP Guidelines and Practical Tips page on the internet]. Sydney: The Royal Australasian College of Physicians. 2012 [cited 2013 May]. Available from URL: http://www.racptelehealth.com .au/guidelines

4 Watts LA, Monk AF, Karnon J, Elshaug AG, Hiller JE. Telemedicine. What happens in remote consultation. Int $J$ Technol Assess Health Care 1999; 15: 220-35.

\author{
5 National Medicare Local Telehealth \\ Support Program. [Telehealth page on the \\ internet]. Canberra: Australian Medicare \\ Local Alliance. 2013 [cited 2013 May]. \\ Available from URL: http:// \\ amlalliance.com.au/ \\ medicare-local-support/telehealth \\ 6 Smith AC, Gray LC. Telemedicine across \\ the ages. Med J Aust 2009; 190: 15-9.
}

